\title{
Persistent Headache Attributed to Traumatic Injury to the Head: Can We Do Better?
}

Keywords: Headache, Head trauma

Headache associated with head injury is poorly understood, and often poorly treated. It can represent a tremendous disability for the patient, even after a relatively mild head injury. In the Alberta Health Services Chronic Pain Center in Calgary, I see patient after patient with debilitating headache which has usually started after a "minor" car accident. I often wonder if we could do more to prevent these terrible life-changing experiences for our patients.

In this issue of the journal, Mares et al. review what is known about the pathophysiology of headache attributed to head trauma, and one of its frequent accompanying symptoms, photophobia. ${ }^{1}$ The authors review the evidence for a number of possible factors that could contribute to this headache syndrome, including microglial activation in the brain parenchyma, dural inflammation related to mast cell degranulation with sensitization of pain pathways, injury to the extra-cranial tissues, direct damage to neuronal brain structures, autoimmune mechanisms, and several others. In the end, as the authors acknowledge, although some progress has been made, the pathophysiology of headache attributed to mild head trauma and the associated photophobia remains to be determined.

Another recent review on the basic mechanisms of "posttraumatic headache" in the journal Headache also came up with no definitive conclusions on the underlying pathophysiology. ${ }^{2}$

Mares et al suggest a general hypothesis that the head injury activates the same mechanisms that lead to migraine or tensiontype headache in patients without trauma. There may be merit to this view, as in the clinic it is often observed that patients with pre-existing migraine have a marked exacerbation of their migraine headaches, in many cases long term, following head trauma.

It may be simplistic to think that we will find a basic underlying mechanism for headache attributed to head trauma. The pathophysiology of this condition may well be complex, with some factors important in some patients and not in others. We may need to learn to subdivide patients with headache after head trauma into subcategories before we can elucidate the underlying mechanisms.

\section{Head Trauma Versus neck Trauma}

Definitions and classification systems are important if we are going to create order out of chaos, and essential for research to advance. A case in point is the loose use of the term "post-traumatic headache." Mares et al. explicitly state that they are using "post-traumatic headache" to describe headache attributed to traumatic injury to the head and are not referring to headache attributed to whiplash. In contrast, a recent review entitled, "An update on the management of post-traumatic headache" dealt mainly with whiplash injuries. ${ }^{3}$ It is important that those who discuss these headache types follow the International Headache Society Classification system (ICHD-3) where possible. ${ }^{4}$ The ICHD-3 classifies persistent headaches attributed to traumatic injury to the head and/or neck into two main categories:

1. Persistent headache attributed to traumatic injury to the head.

2. Persistent headache attributed to whiplash.

Each of these categories has its own diagnostic criteria, and in theory it should be feasible to differentiate which type of "posttraumatic headache" is being discussed. Traumatic injury to the head is clearly defined as "injury resulting from the action of external forces upon the head" and these include "impact between the head and an object, penetration of the head by a foreign body," and "forces generated from blasts or explosions."

It is clearly stated that "trauma due to acceleration/deceleration movements of the head, with flexion/extension of the neck, is classified as whiplash."

In theory this distinction makes sense, in that direct injury to the head might be expected to result in brain changes including glial activation and perhaps inflammation in the dura, while a whiplash injury would be expected to injure upper cervical spine structures (joints, muscles, ligaments, and fascia). These injured cervical structures could, in addition to causing neck pain, refer pain to the head because of the close relationship between the upper cervical nerves and the trigeminal system. ${ }^{5}$ One might also expect, then, that the pathophysiology of these two headache types would be very different, and their management would also be different. Perhaps it is time to abandon the umbrella term "posttraumatic headache."

However, acceleration and deceleration forces on the head without direct head trauma can cause subdural hematomas, for example after roller-coaster rides, ${ }^{6}$ so it might be expected that whiplash could cause trauma to the brain parenchyma as well. Similarly, many injuries to the head may also cause injury to the upper cervical spine. Therefore, it may not be unusual for individual patients to have pathology both in the brain and/or meninges, and in the neck.

In fact, concussion has been defined in part as a traumatic brain injury induced by biomechanical forces resulting from a direct

Received September 3, 2018. Final Revisions Submitted September 10, 2018. Date of ACCEPTANCE SEPTEMBER 11, 2018. 
blow to the head, face, neck, or elsewhere on the body with an impulsive force transmitted to the head. ${ }^{7}$

It can be difficult to sort all this out in the clinic as there are no good objective markers to confirm that trauma to the head is causing the patient's headache. Similarly, although some specific invasive techniques like upper cervical facet blocks can be helpful, there are many potential pain generators in the neck including the atlanto-occipital and atlanto-axial joints that cannot be interrogated reliably by objective techniques in most medical centers.

\section{Prevention}

Where does all this leave us? Mares et al hope their review can be used as a guide for future research, and clearly much more research is needed. At the same time, it behooves all of us who see these patients to become more active in the prevention of head injuries. Some work is already being done, for example, by organizations such as Parachute ${ }^{8}$ and the ThinkFirst National Injury Prevention Foundation. ${ }^{9}$

Much more needs to be done, particularly in the realm of motor vehicle accident prevention. Transport Canada's National Collision Database ${ }^{10}$ reports that in 2015 there were 116,735 motor vehicle collisions in Canada that resulted in personal injury, and in these 161,902 individuals were injured. This amounts on average to 320 injury accidents per day in Canada. Surely we can do better.

\section{Disclosures}

WJB reports grants from Amgen, grants from Allergan, personal fees from Allergan, personal fees from Amgen, personal fees from Novartis, and personal fees from Aralez, outside the submitted work.

\section{Statement of Authorship}

WJB is the author of this article and is solely responsible for its content.

Werner J. Becker

Department of Clinical Neurosciences, University of Calgary,

Calgary, AB, Canada (WJB)

Correspondence to: W. J. Becker, AHS Chronic Pain Centre, 1820 Richmond Road SW, Calgary, AB, Canada T2T $5 C 7$.

Email:wbecker@ucalgary.ca

\section{REFERENCES}

1. Mares C, Dagher JH, Harissi-Dagher M. Narrative review of the pathophysiology of headaches and photosensitivity in mild traumatic brain injury and concussion. Can J Neurol Sci. 2019; 46(1):14-22.

2. Kamins J, Charles A. Posttraumatic headache: basic mechanisms and therapeutic targets. Headache. 2018;58:811-26.

3. Obermann M, Naegel S, Bosche B, Holle D. An update on the management of posttraumatic headache. Ther Adv Neurol Disord. 2015;8(6):311-5.

4. Headache Classification Committee of the International Headache Society. The International Classification of Headache Disorders, 3rd edition. Cephalalgia. 2018;38(1):1-211.

5. Bartsch T, Goadsby PJ. Increased responses in trigeminocervical nociceptive neurons to cervical input after stimulation of the dura mater. Brain. 2003;126(Pt 8):1801-13.

6. Scranton RA, Evans RW, Baskin DS. A motion simulator ride associated with headache and subdural hematoma: first case report. Headache. 2016;56(2):372-8.

7. McCrory P, Feddermann-Demont $\mathrm{N}$, Dvořák $\mathrm{J}$, et al. What is the definition of sports-related concussion: a systematic review. Br J Sports Med. 2017;51(11):877-87.

8. http://www.parachutecanada.org. Accessed September 7, 2018.

9. http://www.thinkfirst.org/concussion. Accessed September 3, 2018.

10. https://www.tc.gc.ca/eng/motorvehiclesafety/tp-tp3322-2015-1487. html. Accessed September 3, 2018. 